\title{
Microscopic response effects in collisions of antiprotons with helium atoms and lithium ions
}

\author{
M. Keim, ${ }^{*}$ A. Achenbach, and H.J. Lüdde \\ Institut für Theoretische Physik, Johann Wolfgang Goethe-Universität, \\ Robert-Mayer-Straße 8, D-60054 Frankfurt, Germany \\ T. Kirchner \\ Max-Planck-Institut für Kernphysik, \\ Saupfercheckweg 1, D-69117 Heidelberg, Germany ${ }^{\dagger}$
}

(Dated: March 27, 2003)

\begin{abstract}
We present total single- and double-ionization cross sections for the collision systems $\bar{p}+\mathrm{He}$ and $\overline{\mathrm{p}}+\mathrm{Li}^{+}$in the $1-1000 \mathrm{keV}$ impact energy range with emphasis on microscopic response effects during the collision. The calculations rely on the basis generator method. In both collision systems the response of the effective electronic interaction to the time-dependent density reduces the ionization cross section at low impact energies significantly. It is shown in comparison with other theories that this reduction is a consequence of time-dependent screening rather than due to dynamical correlation effects.
\end{abstract}

PACS numbers: 34.10.+x; 34.50.-s; 34.50.Fa; 52.20.Hv

\footnotetext{
*electronic address: mkeim@th.physik.uni-frankfurt.de

${ }^{\dagger}$ Present address: Institut für Theoretische Physik, TU Clausthal, Leibnizstraße 10, D-38678 ClausthalZellerfeld, Germany
} 


\section{INTRODUCTION}

Collisions between antiprotons and helium or helium-like ions are dynamical two-electron benchmark systems that are used to examine ionization mechanisms in the presence of electron-electron interaction. In recent years, several nonperturbative theories [1-6] have focused on different mechanisms which might explain the experimental total cross section $[7,8]$ for single-ionization of helium atoms by antiprotons. In this context it is discussed whether time-dependent correlation effects have to be taken into account or whether an appropriate inclusion of the effective single-particle electronic interaction throughout the collision is sufficient to describe this process. At projectile energies below $30-40 \mathrm{keV}$ there is still a considerable discrepancy between different theories and the experiment. In the near future, the ASACUSA collaboration at CERN will have access to a very slow antiproton beam of sufficient intensity to repeat the collision experiments at low collision energies in order to clarify the situation.

We present results for total single- and double-ionization cross sections for collisions of antiprotons with $\mathrm{He}\left(1 \mathrm{~s}^{2},{ }^{1} \mathrm{~S}_{0}\right)$ and $\mathrm{Li}^{+}\left(1 \mathrm{~s}^{2},{ }^{1} \mathrm{~S}_{0}\right)$ based on an effective single-particle description, in which the electron-electron interaction is time-dependent due to microscopic response effects during the collision. In the collision system $\bar{p}+$ He the maximum of the experimental results for single ionization is very well reproduced by our calculated cross section. In contrast, the recently published results of Tong et al. [6] underestimate this maximum by approximately 16\%, although the same level of approximation was used. Atomic units $\left(\hbar=m_{e}=e=1\right)$ are used throughout.

\section{THEORY}

Within the framework of time-dependent density functional theory (TDDFT) $[9,10]$ the $N$-electron system is represented by a set of single-particle equations

$$
i \partial_{t} \psi_{j}(\mathbf{r}, t)=\hat{h}(t) \psi_{j}(\mathbf{r}, t), j=1, \ldots, N
$$

with the Hamiltonian

$$
\hat{h}(t)=-\frac{1}{2} \Delta-\frac{Q_{T}}{r}-\frac{Q_{P}}{|\mathbf{r}-\mathbf{R}(t)|}+v_{e e}([n(t)], \mathbf{r}, t)
$$


and the effective potential $v_{e e}$ due to the electron-electron interaction. $v_{e e}$ is a unique functional of the one-particle density which is exactly given by

$$
n(\mathbf{r}, t)=\sum_{j=1}^{N}\left|\psi_{j}(\mathbf{r}, t)\right|^{2} .
$$

$Q_{T}$ and $Q_{P}$ denote the charges of target and projectile nuclei. We assume that the projectile is moving on a straight line trajectory $\mathbf{R}(t)=(b, 0, v t)$ with impact parameter $b$ and constant velocity $v$. This so-called Kohn-Sham scheme $(1,2,3)$ is an exact mapping of the timedependent many-electron system on an effective single-particle description. In the present case of a two-electron spin-singlet system only one orbital $\psi_{j}$ has to be propagated, and the density is given by $n(\mathbf{r}, t)=2|\psi(\mathbf{r}, t)|^{2}$.

To solve the time-dependent single-particle equations (1) we use a basis representation obtained from the basis generator method (BGM) [11-15]

$$
\begin{aligned}
\psi(\mathbf{r}, t) & =\sum_{K=0}^{L} \sum_{J=0}^{M} \sum_{k=1}^{N} c_{k}^{K J}(t) \chi_{k}^{K J}(\mathbf{r}, t) \\
\chi_{k}^{K J}(\mathbf{r}, t) & =W_{\mathrm{T}}\left(r, \epsilon_{\mathrm{T}}\right)^{K} W_{\mathrm{P}}\left(\mathbf{r}, t, \epsilon_{\mathrm{P}}\right)^{J} \chi_{k}^{00}(\mathbf{r}) \\
W_{\mathrm{T}}\left(r, \epsilon_{\mathrm{T}}\right) & =\frac{1-\exp \left(-\epsilon_{\mathrm{T}} r\right)}{r} \\
W_{\mathrm{P}}\left(\mathbf{r}, t, \epsilon_{\mathrm{P}}\right) & =\frac{1-\exp \left(-\epsilon_{\mathrm{P}}|\mathbf{r}-\mathbf{R}(t)|\right)}{|\mathbf{r}-\mathbf{R}(t)|},
\end{aligned}
$$

with $W_{P / T}$ denoting the regularized Coulomb interactions with respect to the projectile and target center. The set of bound eigenfunctions of the undisturbed helium atom $\chi_{k}^{00}(\mathbf{r})$ accounts for the elastic and target excitation channels, while the set of pseudostates $\chi_{k}^{K J}(\mathbf{r})$ describes ionization and capture. Since the capture channel is closed in the case of antiproton impact and the continuum contribution is assumed to be primarily centered around the target, a hierarchy of pseudo-states based on the regularized target potential (4c) was found to be sufficient (see, e.g., calculations for $\bar{p}+\mathrm{H}$ collisions $[16,17])$. Hence we set $M=0$ in the present calculations.

\section{A. The representation of $v_{e e}$}

The exchange-only (x-only) approximation of the electronic potential $v_{e e}$ for the spinsinglet configuration of the helium-like target yields

$$
v_{e e}(\mathbf{r}, t)=\frac{1}{2} \int \frac{n\left(\mathbf{r}^{\prime}, t\right)}{\left|\mathbf{r}-\mathbf{r}^{\prime}\right|} d^{3} r^{\prime}
$$


$v_{e e}$ obviously fulfils the Poisson equation

$$
\Delta v_{e e}(\mathbf{r}, t)=-\frac{1}{2} 4 \pi n(\mathbf{r}, t)
$$

For the evaluation of the effective potential, which describes the electron-electron interaction during the time-propagation, we make the ansatz

$$
v_{e e}(\mathbf{r}, t)=\sum_{\lambda=1}^{\Lambda} \sum_{l=0}^{D} \sum_{m=-l}^{l} d_{l m}^{\lambda}(t) W_{\mathrm{T}}\left(r, \epsilon_{\mathrm{T}}\right)^{\lambda} Y_{l m}(\Omega)
$$

The coefficients $d_{l m}^{\lambda}(t)$ are determined by solving Eq. (6) in each timestep. The quality of the approach (7) with the parameters $\Lambda$ and $D$ is examined in two ways.

(i) We compare the representation (7) with the exact x-only groundstate potential of helium $[18,19]$. In this case, the relative deviation between the ansatz (7) with $\Lambda=6$ and $D=0$ and the exact x-only potential is less than $1 \%$ as illustrated in Fig. 1. Moreover, Table I shows that the eigenvalues of the $K-, L$-, and $M$-shell orbitals of the helium groundstate configuration are reproduced to a few microhartree.

(ii) The ansatz (7) is further examined by comparing the adiabatic single-particle groundstate energy of the quasimolecule $(\overline{\mathrm{p}} \mathrm{He})(\Lambda=6, D=0)$ with the results of an exact x-only calculation [19]. Figure 2 shows that over the entire $R$-range a very high accuracy is achieved. We note that in the case of a spin-singlet system the x-only limit of DFT is identical with the Hartree-Fock (HF) limit. In particular, the well known HF single-particle energies for the negative $H^{-}$ion $\left(\epsilon_{H^{-}}=-0.046223\right.$ a.u. $)$ and the He atom $\left(\epsilon_{H e}=-0.9179\right.$ a.u. $)$ are approached at small and large internuclear distances, respectively. It is noteworthy that in the case of a no-response approximation, where the effective electron-electron potential is frozen to the initial groundstate configuration $\left(v_{e e}(\mathbf{r}, t)=v_{e e}^{0}(r)\right)$, the $H^{-}$-formation is not feasible since the corresponding eigenvalue for small $\mathrm{R}$ becomes positive (solid line in Fig. 2).

\section{B. Extraction of probabilities}

In order to facilitate the interpretation of the final wave function we have orthogonalized the set of pseudostates $\left\{\chi_{k}^{K 0}(\mathbf{r}), K>0\right\}$ with respect to the generating basis $\left\{\chi_{k}^{00}(\mathbf{r})\right\}$. Therefore, the coefficients $c_{k}^{00}(t)$ can be associated with probability amplitudes for targetexcitation, while the total single-particle ionization probability is deduced from the normal- 


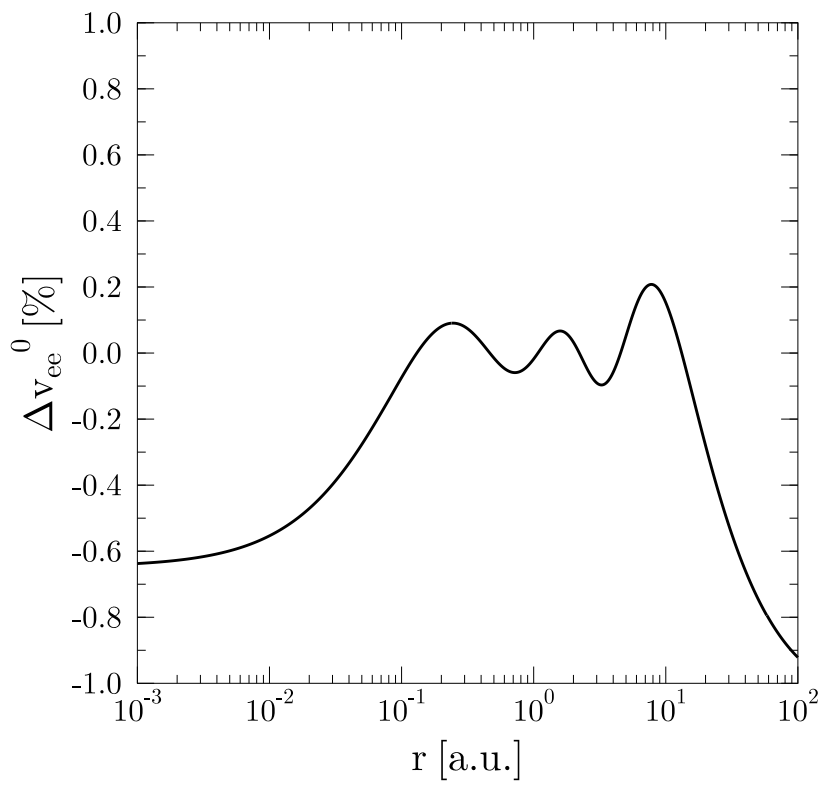

FIG. 1: Relative error of $v_{e e}$ obtained with expansion $(7)(\Lambda=6, D=0)$ with respect to the exact $\mathrm{x}$-only approximation of the helium groundstate $[18,19]$.

TABLE I: Eigenvalues of K-,L- and M-shell orbitals of the helium groundstate configuration in hartree, obtained with expansion $(7)(\Lambda=6, D=0)$ and the exact x-only approximation $[18,19]$.

\begin{tabular}{ccc}
\hline \hline Orbital & exact x-only $[18,19]$ & Model $(7)$ \\
\hline $1 s$ & -0.91796 & -0.91796 \\
$2 s$ & -0.15833 & -0.15835 \\
$2 p_{0, \pm 1}$ & -0.12743 & -0.12746 \\
$3 s$ & -0.06466 & -0.06468 \\
$3 p_{0, \pm 1}$ & -0.05636 & -0.05637 \\
$3 d_{0, \pm 1, \pm 2}$ & -0.05557 & -0.05567 \\
\hline \hline
\end{tabular}

ization of the final wave function

$$
p_{\text {ion }}=1-\sum_{k=1}^{N}\left|\left\langle\chi_{k}^{00} \mid \psi(t)\right\rangle\right|^{2} .
$$

The probabilities for emitting one or two electrons are then calculated by the usual binomial formulae

$$
\begin{aligned}
& P_{1}=2 p_{\text {ion }}\left(1-p_{\text {ion }}\right) \\
& P_{2}=p_{\text {ion }}^{2}
\end{aligned}
$$




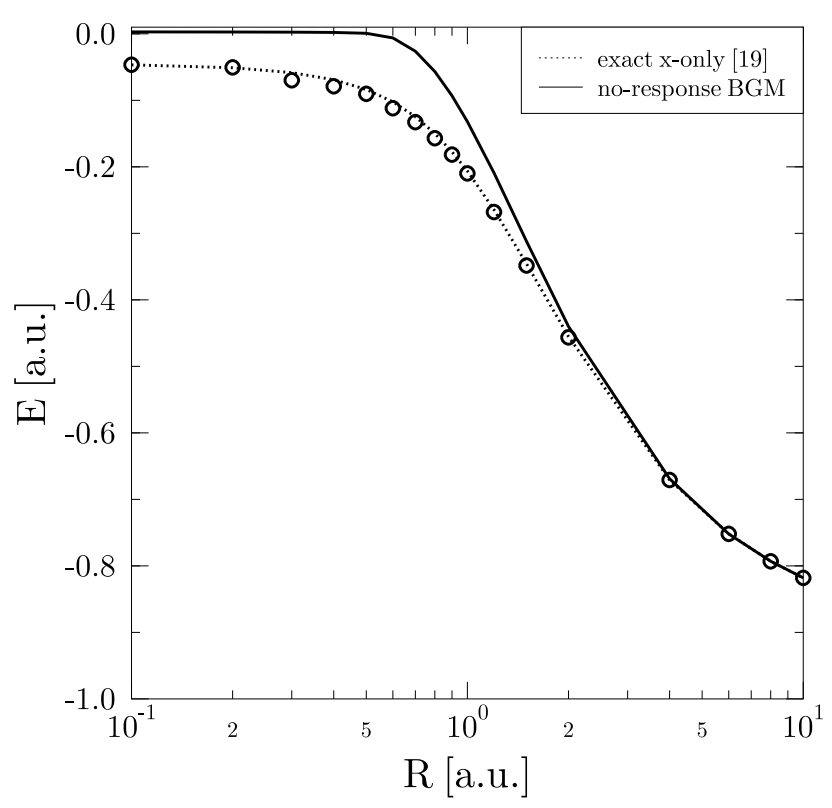

FIG. 2: Adiabatic single-particle groundstate energy level of the quasimolecule $(\overline{\mathrm{p}} \mathrm{He})$ calculated with: dotted line, exact x-only [19]; solid line, no response BGM; (०) ansatz (7) with parameters $\Lambda=6$ and $D=0$.

Moreover, a net ionization probability $P_{\text {net }}$ can be defined as

$$
P_{\text {net }}=P_{1}+2 P_{2}=2 p_{\text {ion }}
$$

giving the average number of emitted electrons. While $P_{\text {net }}$ is exact on the basis of DFT, the expressions for single and double ionization (9) and (10) are not. In fact, as we will argue below it is the binomial approach to the two-electron observable $P_{2}$ that is responsible for the discrepancies observed in Fig. 5 and not the time-propagation of the collision system (for a detailed discussion see Ref. [10]).

The analysis (8) with respect to the generating basis $\left\{\left|\chi_{k}^{00}\right\rangle\right\}$ would be exact if the initial and final Hamiltonian of the collision system were identical. In fact, this is not the case if time-dependent response is considered in the effective potential. As $v_{e e}$ is a linear functional of the time-dependent density one obtaines from Eq. (5)

$$
v_{e e}^{0}(r)=\lim _{t \rightarrow-\infty} v_{e e}(\mathbf{r}, t) \neq \lim _{t \rightarrow+\infty} v_{e e}(\mathbf{r}, t)
$$

It is then straightforward to show that the coefficients $c_{k}^{00}(t)=\left\langle\chi_{k}^{00} \mid \psi(t)\right\rangle$ will never converge in time due to the potential difference $v_{e e}(\mathbf{r}, t)-v_{e e}^{0}(r)$ (see the collision history in Fig. 7). 
This problem is well known in literature and has been discussed over many years (e.g., $[20,21])$.

In accordance with the boundary conditions of the collision system one has to define a new set of final states $\left\{\left|\varphi_{k}^{0}\right\rangle\right\}$ which are solutions of the asymptotic form of Eq. (1). It has been shown in Ref. [22] that eigenfunctions of the asymptotic time-dependent Hamiltonian

$$
\hat{h}(t)\left|\varphi_{k}^{0}(t)\right\rangle=\epsilon_{k}(t)\left|\varphi_{k}^{0}(t)\right\rangle
$$

present a reasonable approach to the exact final states. As the energies depend on time only parametrically these states are correlated in form of a dynamic correlation diagram. Substituting $\left\{\left|\varphi_{k}^{0}\right\rangle\right\}$ for $\left\{\left|\chi_{k}^{00}\right\rangle\right\}$ in Eq. (8) one obtains the full lines in Fig. 7, which will be further discussed in Sec. III C. Since the states $\left\{\left|\varphi_{k}^{0}\right\rangle\right\}$ do not exactly fulfil the formally correct boundary conditions of the collision system, the probabilities are still fluctuating, though, on a much smaller scale than the probabilities calculated with $\left\{\left|\chi_{k}^{00}\right\rangle\right\}$. It has also been shown in Ref. [22] that these small fluctuations are due to the explicit time-dependence of the asymptotic potential, $\left\langle\varphi_{i}^{0}(t)\left|\dot{v}_{e e}(t)\right| \varphi_{j}^{0}(t)\right\rangle$.

\section{RESULTS}

For the investigated collision systems $\left(\overline{\mathrm{p}}+\mathrm{He}\right.$ and $\left.\overline{\mathrm{p}}+\mathrm{Li}^{+}\right)$, we select 107 states from a BGM basis set (4b) with $N=20$ (i.e., excited states up to $n=4$ are exactly represented), $M=0$, and $L=8$. The expansion of the effective electron-electron interaction potential (7) is limited to $\Lambda=6$ and $D=2$.

\section{A. $\overline{\mathbf{p}}+\mathbf{H e}$}

In Figs. 3 and 4 several theoretical results for single ionization are compared with experimental data. The theoretical data are combined into two groups: on the one hand, those results without correlation but with different levels of approximation of the effective singleparticle potential $v_{e e}$ (Fig. 3), and on the other hand those results that include dynamical correlation (Fig. 4). Within the no-response approximation $\left(v_{e e}(\mathbf{r}, t)=v_{e e}^{0}(r)\right)$ the electronelectron potential is frozen to its initial ground state configuration. This model leads to an increasing overestimation of ionization with decreasing impact energy. This problem is 
partly remedied by the inclusion of an adiabatic response potential [5], denoted as $v_{\bmod }$ in Figs. 3 and 5

$$
v_{\text {mod }}=-a(R) \exp (-r)
$$

where the parameter $a(R)$ is chosen such that the binding energy of the lowest single-particle level reproduces the ionization potential of the quasimolecular system $(\overline{\mathrm{p}} \mathrm{He})$ as a function of $R$. In this way, the model accounts for the adiabatic stabilization of the electrons in the two-center Coulomb field, i.e., it enables the formation of $\mathrm{H}^{-}$ions.

The best result, however, is obtained if the potential includes the full microscopic x-only response due to the time propagation of the electronic density. In this case, not only are variations of the binding energy but also polarization effects during the collision taken into account.

Of even greater importance is the comparison between the results of the family of theories including dynamical correlation and the present effective single particle calculation including microscopic response (Fig. 4). AO1 [3] and AO2 [4] are atomic orbital coupled-channel results, using different single-centered expansions for the two-electron wave function. Multicut FIM [1] denotes the so called multi-cut forced-impulse method, in which the electronelectron interaction is turned on at a few discrete time-steps during the propagation of the electronic wave function, and MEHC is the multi-electron hidden crossing method of Ref. [2]. As one can see in Fig. 4 the present calculation shows a much better agreement with the experimental data even at lower impact energies. This clearly indicates that single ionization does not depend on time-dependent correlation effects, but certainly depends on an appropriate inclusion of response effects.

It is interesting to compare the present BGM data for single ionization with the results of Ref. [6], which rely also on a single-particle calculation with a microscopic potential. The results of Ref. [6] underestimate the experimental data by approximately $16 \%$ around the peak position of the single-ionization cross section whereas our calculations agree quite well with the experimental results. In addition, the slope of the curve for total single ionization taken from Ref. [6] is considerably less steep in the low-energy regime compared to our present BGM results with the intersection point of both curves at $E_{P}=5 \mathrm{keV}$. It is, however, shown in Fig. 4 that for small impact energies our calculation approaches remarkably well the MEHC-curve [2], which is supposed to represent the correct adiabatic limit. Given that both the BGM and the time-propagation method used in Ref. [6] proved 
their convergence for many other problems, e.g., for $\bar{p}+\mathrm{H}$ collisions, we conclude that the deviations observed in Fig. 3 mirror deficiencies of the response potential used in Ref. [6]. In that paper it was argued that self-interaction corrections were taken into account in $v_{e e}$ but the final form of the potential was not given explicitly. We emphasize that the x-only potential (5) used to calculate the present data is exact, i.e., completely self-interaction free. It is interesting that the total single-ionization cross section is rather sensitive to the exact treatment of time-dependent screening and exchange effects.

Figure 5 shows the results obtained for double ionization in comparison with other theories and experiment. In contrast to the no-response calculation and the calculation with an adiabatic response potential [5], the results with microscopic response show a further reduction of the cross section in the range of small to intermediate collision energies. However, in this range a considerable overestimation of the experimental data persists. As mentioned previously, this overestimation of the cross section for double ionization is mainly caused by the binomial approximation of the observable $P_{2}$. Using (9) and (10) one finds a relation between $P_{1}$ and $P_{2}$

$$
P_{1}=2\left(\sqrt{P_{2}}-P_{2}\right)
$$

This relation is obviously an artifact of the binomial analysis, as single and double ionisation must be independent processes and can not be related. The problem with (15) becomes obvious as the relation allows a maximum of 0.5 for $P_{1}$ whereas $P_{2}$ can approach 1 . This shows that the extraction of many-particle observables should be interpreted carefully if calculated within a single-particle approximation.

\section{B. $\overline{\mathbf{p}}+\mathbf{L} \mathbf{i}^{+}$}

In comparison with the more weakly bound He target, the total cross section for single ionization in $\overline{\mathrm{p}}+\mathrm{Li}^{+}$collisions is much smaller and its maximum is shifted to higher impact energies (Fig. 6). This corresponds to the different characteristics of the active electron and simply reflects the scaling properties of the Schrödinger equation. Comparable to the collision system $\bar{p}+\mathrm{He}$, the cross sections of the no-response and the response cases coincide at high impact energies since the electronic cloud has not enough time to adapt to the rapidly changing two-center-potential of the nuclei. At the other extreme, i.e., at very small

impact energies, both curves approach zero in the collision system $\bar{p}+\mathrm{Li}^{+}$because the 


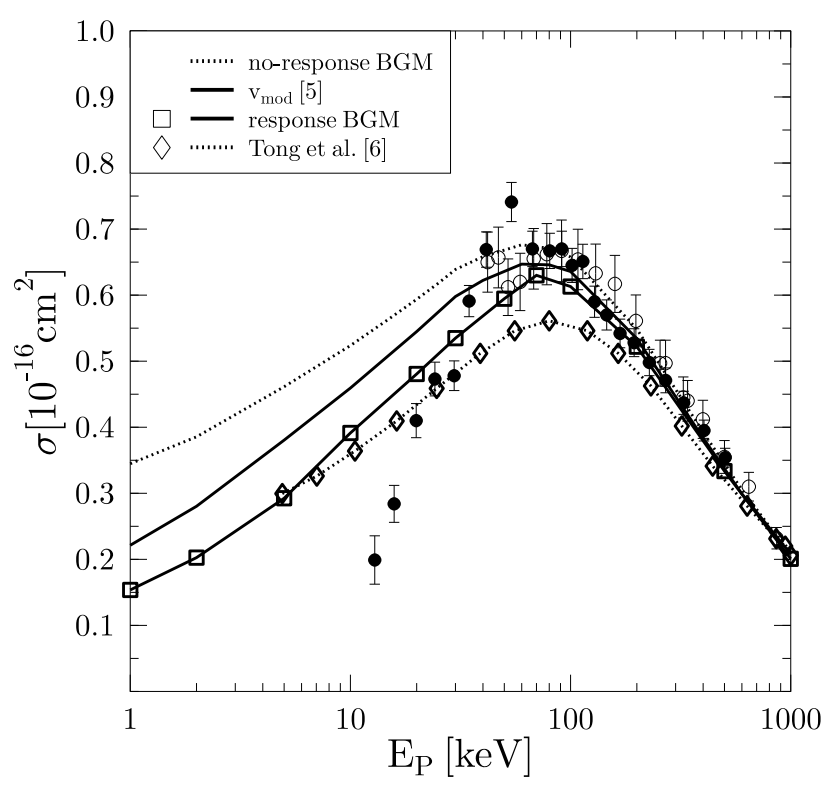

FIG. 3: Total cross section for single ionization as a function of impact energy for $\overline{\mathrm{p}}+$ He collisions in comparison with other one-electron theories. Experiment: (०) [7], (•) [8].

quasi-molecule $\left(\overline{\mathrm{p}} \mathrm{Li}^{+}\right)$is bound even without response in the adiabatic limit. This is a consequence of the higher charge of the target nucleus. The fact that response effects are comparable in both cases $\left(\mathrm{He}\right.$ and $\mathrm{Li}^{+}$) is nevertheless striking. One might expect that the stronger electron-nucleus interaction overbalances the electronic potential for the ionic target. However, this is not true: at $E_{P}=10 \mathrm{keV}$, for instance, response effects suppress the single-ionization cross section by $25 \%$ for $\mathrm{He}$ and by $30 \%$ for the $\mathrm{Li}^{+}$target atom.

\section{Ionization mechanism}

Figure 7 depicts the evolutions of the probabilities of the elastic channel (upper curves) and the net ionization $P_{\text {net }}$, scaled by a factor of 0.5 (lower curves), calculated in terms of the eigenfunctions $\left\{\left|\chi_{k}^{00}\right\rangle\right\}$ (dotted lines) and $\left\{\left|\varphi_{k}^{0}\right\rangle\right\}$ (solid lines), respectively. As the eigenfunctions $\left\{\left|\varphi_{k}^{0}\right\rangle\right\}$ of the full time-dependent Hamiltonian (including response) reflect the momentary state of the collision system to some extent, the corresponding occupation numbers have more physical meaning than those obtained from the analysis with respect to the undisturbed initial states $\left\{\left|\chi_{k}^{00}\right\rangle\right\}$. Except for the fluctuations which have been discussed in Sec. IIB, two main features can be observed in Fig. 7: (i) a rapid decline of the elastic channel accompanied by an increase of ionization around the closest approach followed by 


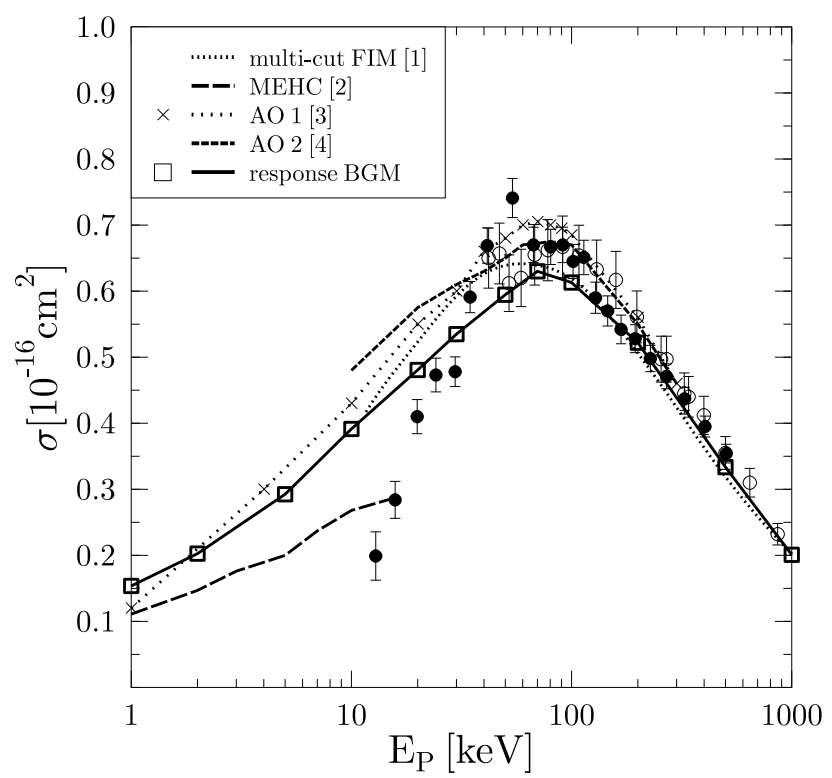

FIG. 4: Total cross section for single ionization as a function of impact energy for $\bar{p}+$ He collisions in comparison with other two-electron theories. Multi-cut FIM [1], MEHC [2], AO1 [3], and AO2 [4]. Experiment: (o) [7], (•) [8].

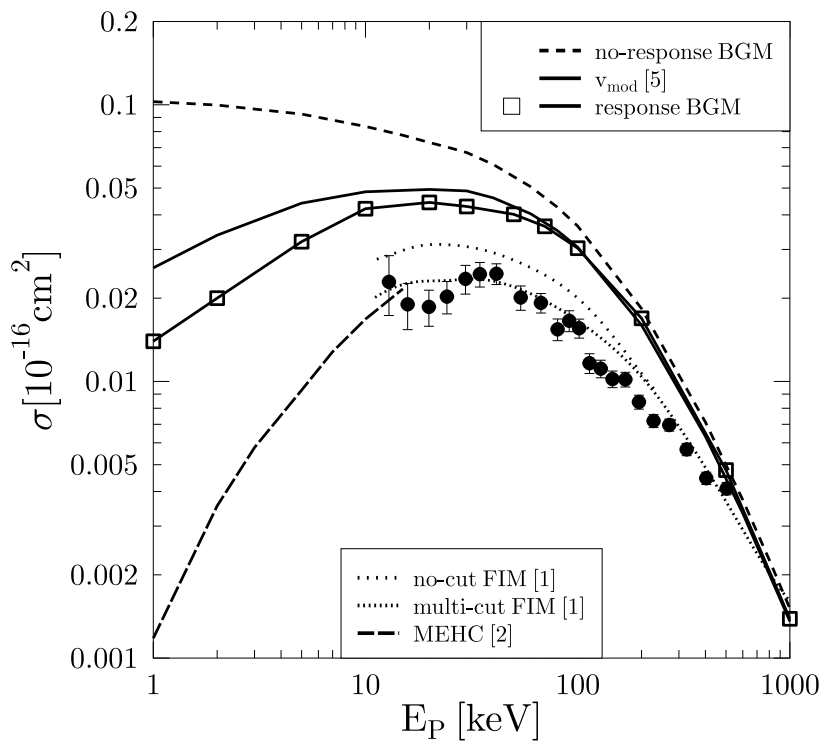

FIG. 5: Total cross section for double ionization as a function of impact energy for $\overline{\mathrm{p}}+$ He collisions in comparison with other theories. In the multi-cut FIM calculation dynamical electron-electron correlation is included, whereas in the no-cut FIM calculation electron-electron correlation is only considered in the initial and the final states. Experiment: (o) [7], (•) [8]. 


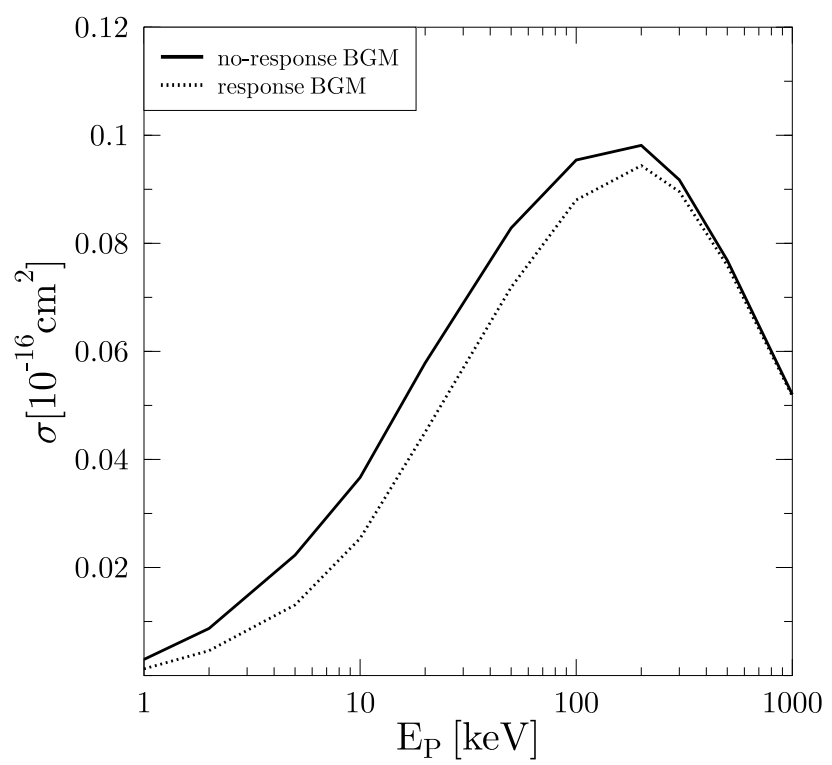

FIG. 6: Total cross section for single ionization as a function of impact energy for $\overline{\mathrm{p}}+\mathrm{Li}^{+}$collisions with and without microscopic response.

(ii) a relaxation of the ionization probability in contrast to an immediate stabilization of the elastic channel. The first feature can be attributed to some form of target eclipse: In collisions with impact parameters around the $r$-expectation value of the active electron (which make the main contribution to the total cross section) the charge of the approaching antiproton partially screens the target core. This leads to a reduction of its attraction and gives the electronic cloud the possibility to escape. When the antiproton is leaving the inner region of the electronic cloud, the attraction of the target core sets in again and the primary ionization process is stopped immediately. The relaxation phenomenon of the ionization probability around $2.5<z<15$ a.u. is due to recapture of the electron(s) into low lying excited states of the He target.

\section{CONCLUSIONS}

In this paper, the role of microscopic response effects in ion-atom collisions with two active electrons has been examined within a single-electron description on the basis of timedependent density functional theory. We have used the x-only approximation of the effective potential to describe the electron-electron interaction. The convergence of the ansatz used for this potential was demonstrated by considering the adiabatic situations for atomic helium 


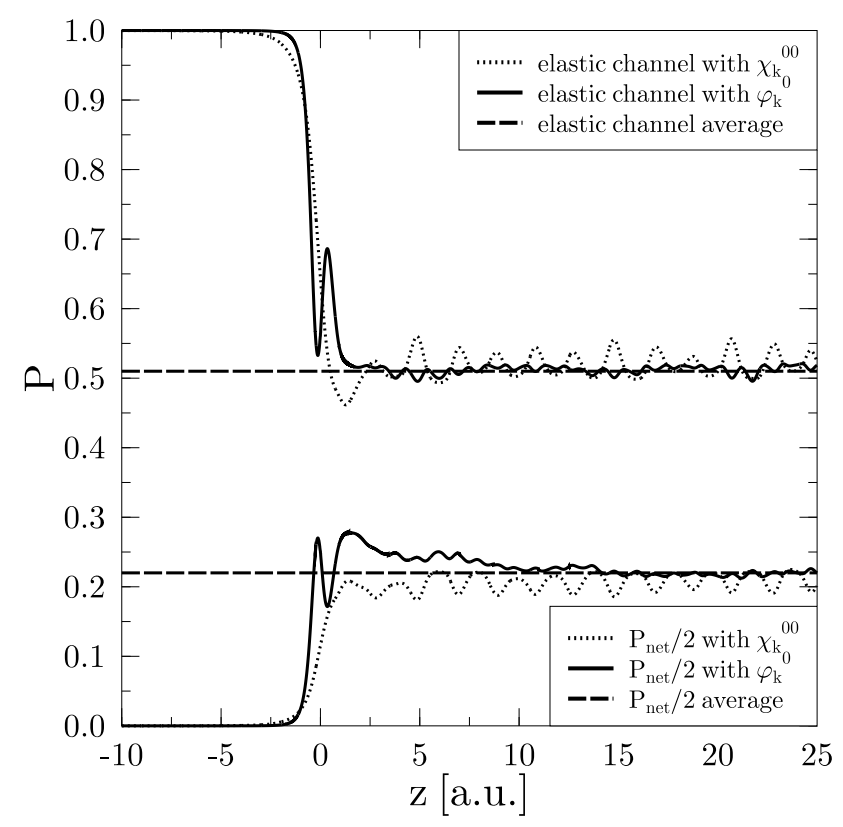

FIG. 7: Time evolution $(z=v t)$ of $P_{\text {net }} / 2$ and the elastic channel for the collision system $\overline{\mathrm{p}}+$ He at $E_{P}=5 \mathrm{keV}$ and $b=0.5$ a.u., obtained with different choices of eigenfunctions: dotted lines, eigenfunctions $\left\{\left|\chi_{k}^{00}\right\rangle\right\}$; solid lines, eigenfunctions $\left\{\left|\varphi_{k}^{0}\right\rangle\right\}$; upper curves, elastic channel; lower curves, $P_{\text {net }}$; dashed horizontal lines, average values for $z \rightarrow \infty$.

and the quasimolecule $(\overline{\mathrm{p}} \mathrm{He})$. The calculated cross section for single ionization in the collision system $\overline{\mathrm{p}}+$ He reproduces the maximum of the experimental data at about $E_{P}=70 \mathrm{keV}$ very well, whereas the calculation of Ref. [6], which is based on similar concepts, underestimates this cross section by approximately $16 \%$. At very small collision energies our calculated cross section approaches the results of the MEHC-calculation [2], which is supposed to reproduce the correct low-energy limit. From these findings we overall conclude that it is essential that the calculation accounts for exchange and screening effects on a microscopic level. It has been shown that the ionization mechanism can be described by a kind of target eclipse. Our results for the collision system $\bar{p}+\mathrm{Li}^{+}$show that even in this case the cross section for single ionization is strongly reduced at low collision energies by microscopic response effects.

\section{Acknowledgments}

We would like to thank E. Engel for providing us with the atomic structure calculations. One of us (T.K.) gratefully acknowledges support by the Leibniz Programm of the Deutsche 
Forschungsgemeinschaft.

[1] T. Bronk, J. F. Reading, and A. L. Ford, J. Phys. B 31, 2477 (1998).

[2] G. Bent, P. S. Krstíc, and D. R. Schultz, J. Chem. Phys. 108, 1459 (1998).

[3] T. G. Lee, H. C. Tseng, and C. D. Lin, Phys. Rev. A 61, 062713 (2000).

[4] A. Igarashi, A. Ohsaki, and S. Nakazaki, Phys. Rev. A 62, 052722 (2000).

[5] T. Kirchner, M. Horbatsch, E. Wagner, and H. J. Lüdde, J. Phys. B 35, 925 (2002).

[6] X. M. Tong, T. Watanabe, D. Kato, and S. Ohtani, Phys. Rev. A 66, 032709 (2002).

[7] L. H. Andersen, P. Hvelplund, H. Knudsen, and S. P. Møller, Phys. Rev. Lett. 57, 2147 (1986).

[8] P. Hvelplund, H. Knudsen, U. Mikkelsen, E. Morenzoni, S. P. Møller, E. Uggerhøj, and T. Worm, J. Phys. B 27, 925 (1994).

[9] E. K. U. Gross, J. F. Dobson, and M. Petersilka, in Topics in Current Chemistry, edited by R. F. Nalewajski (Springer, Heidelberg, 1996), vol. 181, p. 81.

[10] H. J. Lüdde, in Many-Particle quantum dynamics in atomic and molecular fragmentation, edited by V. P. Shevelko and J. Ullrich (Springer, Heidelberg, 2003).

[11] H. J. Lüdde, A. Henne, T. Kirchner, and R. M. Dreizler, J. Phys. B 29, 4423 (1996).

[12] O. J. Kroneisen, H. J. Lüdde, T. Kirchner, and R. M. Dreizler, J. Phys. A 32, 2141 (1999).

[13] T. Kirchner, H. J. Lüdde, M. Horbatsch, and R. M. Dreizler, Phys. Rev. A 61, 052710 (2000).

[14] T. Kirchner, M. Keim, A. Achenbach, H. J. Lüdde, O. J. Kroneisen, and R. M. Dreizler, Phys. Scr. T80B, 270 (1999).

[15] D. Elizaga, L. F. Errea, J. D. Gorfinkiel, C. Illescas, L. Méndez, A. Macías, A. Riera, A. Rojas, O. J. Kroneisen, T. Kirchner, et al., J. Phys. B 32, 857 (1999).

[16] T. Kirchner, H. J. Lüdde, O. J. Kroneisen, and R. M. Dreizler, Nucl. Instrum. Methods Phys. Res. B 154, 46 (1999).

[17] M. Keim, A. Achenbach, H. J. Lüdde, and R. M. Dreizler, XXII. ICPEAC Santa Fe, Abstracts of Contributed Papers p. 413 (2001).

[18] E. Engel and S. H. Vosko, Phys. Rev. A 47, 2800 (1993).

[19] E. Engel, private communication. 
[20] W. Stich, H. J. Lüdde, and R. M. Dreizler, Phys. Lett. 99A, 41 (1983).

[21] K. Gramlich, N. Grün, and W. Scheid, J. Phys. B 19, 1457 (1986).

[22] T. Kirchner, M. Horbatsch, H. J. Lüdde, and R. M. Dreizler, Phys. Rev. A 62, 042704 (2000). 